\title{
Clinical study of placenta previa and its effect on maternal health and fetal outcome
}

\author{
Sarojini*, Malini K. V., Radhika
}

Department of Obstetrics and Gynaecology, Bangalore Medical College and Research Institute, Bangalore, Karnataka, India

Received: 01 August 2016

Accepted: 09 September 2016

\section{*Correspondence:}

Dr. Sarojini,

E-mail: prabhusaroj@gmail.com,

Copyright: (c) the author(s), publisher and licensee Medip Academy. This is an open-access article distributed under the terms of the Creative Commons Attribution Non-Commercial License, which permits unrestricted non-commercial use, distribution, and reproduction in any medium, provided the original work is properly cited.

\begin{abstract}
Background: When the placenta is implanted partially or completely in the lower uterine segment it is called placenta previa. The objective of the study was to determine the incidence, obstetric risk factors, obstetric management, maternal mortality and morbidity, perinatal outcome in women presenting with placenta previa.

Methods: Total 106 pregnant women with placenta previa were analyzed between January to December 2015. After applying the inclusion and exclusion criteria these women were analyzed with respect to their age, parity, gestational age and clinical features at presentation, history of warning bleeding, duration of hospitalization, need for blood transfusion, period of gestation at delivery, route of delivery and ICU admissions. For the newborn APGAR score, birth weight, need for NICU admission, still birth rate, neonatal mortality rate are noted down.

Results: In this study $0.64 \%$ of the deliveries were complicated with placenta previa among them $23.6 \%$ women were above 30 years of age and $80.2 \%$ were multigravidas. $60.4 \%$ had major degree placenta previa, $36.8 \%$ had prior cesarean deliveries, $7.5 \%$ had prior abortion, $39.7 \%$ preterm deliveries. $85.8 \%$ cases delivered by cesarean delivery, $12.7 \%$ cases had postpartum haemorrhage and $4.7 \%$ had adherent placenta. There were $86.8 \%$ ICU admissions, $3.8 \%$ cases of acute kidney injury in present series.

Conclusions: Advancing maternal age, multiparity, prior cesarean section, and prior abortions are independent risk factors for placenta previa. Placenta praevia remains a risk factor for adverse maternal and perinatal outcome. The detection of placenta previa should encourage a careful evaluation with timely delivery to reduce the associated maternal and perinatal complications.
\end{abstract}

Keywords: Placenta previa, Prior cesarean delivery, Adherent placenta, Postpartum haemorrhage, Maternal morbidity

\section{INTRODUCTION}

When the placenta is implanted partially or completely in the lower uterine segment it is called placenta previa. About one third of the ante partum haemorrhage belongs to placenta previa. The most characteristic event in placenta previa is painless haemorrhage, which usually does not appear until near the end of the second trimester or after. ${ }^{1}$ The classical features of bleeding in placenta previa are sudden onset, painless, apparently causeless and recurrent. It is associated with increased maternal morbidity and mortality due to increased incidence of hemorrhagic shock, increased operative interventions and sepsis. There is higher incidence of perinatal mortality and morbidity due to preterm delivery and its related complications like low birth weight, birth asphyxia and neonatal sepsis.

The incidence of placenta previa is around 1 in 300 deliveries. ${ }^{2}$ Advancing maternal age increases the risk of placenta previa. At the extremes it is 1 in 1500 for women 19 years of age or younger and it is 1 in 100 for 
women older than 35 years of age. ${ }^{3}$ Multiparity is associated with previa. Prior cesarean delivery increases the likelihood of placenta previa. ${ }^{4}$ Incidence increases from $1.9 \%$ with 2 prior cesareans to $4.1 \%$ with 3 or more. ${ }^{5}$ The simplest, most precise and safest method of placental localization is provided by transabdominal sonography. ${ }^{1}$ There is increased incidence of ante partum hemorrhage leading to maternal shock and its consequences, increased incidence of operative interventions, increased incidence of postpartum hemorrhage all posing increased risk of maternal morbidity and mortality. ${ }^{6}$

Preterm delivery is the major cause of perinatal death even with expectant management of placenta previa. ${ }^{7}$ Although some investigators suggested that congenital malformations are increased with previa, crane and coworkers were the first to confirm this. ${ }^{3}$ For reasons that are unclear, in cases of placenta previa fetal anomalies were increased 2.5 fold. ${ }^{8,9}$ Management of placenta previa depends on presentation, gestational age and degree of previa. ${ }^{6,10}$ When mothers life is not at risk, expectant management will improve the outcome. ${ }^{11}$

\section{METHODS}

A prospective observational study conducted at Vanivilas hospital between January to December 2015 for 12 months. Objective of this study is to determine the incidence, demographic features, obstetric risk factors, obstetric management, maternal mortality and morbidity, perinatal outcome in women presenting with placenta previa.

All 106 women who's gestational age is beyond 28 weeks and who are diagnosed with placenta previa at or after admission and during cesarean delivery are included in the study. Details of their age, parity, gestational age \& clinical features at presentation, detailed history of current pregnancy and previous pregnancies, period of gestation at which placenta previa was diagnosed, history of warning bleeding etc are documented. Women are subjected to a detailed clinical examination. Duration of hospitalization, need for blood transfusion, period of gestation at delivery, route of delivery (vaginal or cesarean), need for extra surgical maneuvers during operative delivery to prevent or to stop bleeding like cervico-isthmic stich, uterine artery ligation, stepwise devascularisation and hysterectomy and need for ICU admissions are noted down. An analysis of maternal mortality and morbidity was done with respect to development of hypovolemic shock, DIC, anemia, acute kidney injury, septicemia and maternal deaths.

For the newborn gestational age at delivery, APGAR score, birth weight, need for NICU admission, still birth rate, neonatal mortality rate, presence of congenital anomalies are noted down. Both mother and baby are followed up throughout the period of their hospitalization till discharge.

\section{RESULTS}

The following data was obtained from the present study. During the study period, there were 16361 deliveries, of which, $0.64 \%$ were complicated with placenta previa.

The age distribution of present study group is shown in Table 1 . Nearly one fourth of women are above 30 years of age.

\section{Table 1: Age distribution.}

\begin{tabular}{|lll|}
\hline Age & Number & Percentage \\
\hline$<20$ years & 10 & 9.4 \\
\hline 20-24 years & 32 & 30.2 \\
\hline 25-29 years & 39 & 36.8 \\
\hline 30 years and above & 25 & 23.6 \\
\hline
\end{tabular}

In the present series there are $21(19.8 \%)$ primis, 25 $(23.6 \%)$ second gravidas, $35(33 \%)$ third gravidas, 24 $(22.7 \%)$ fourth gravidas and $1(0.9 \%)$ fifth gravida. More than three fourth of women $(80.2 \%)$ in this study are multigravidas.

Type of placenta previa depending the location (noted either by placental localization by ultrasound or previa noted during cesarean delivery for some other indication and where ultrasound examination had failed to notice placenta previa) is shown in Table 2. There were 64 $(60.4 \%)$ cases of major degree placenta previa in the present series.

Table 2: Location of placenta (by USG and intraop findings).

\begin{tabular}{|lllll|}
\hline $\begin{array}{l}\text { Type of } \\
\text { placenta }\end{array}$ & Number & \multicolumn{3}{l|}{ Percentage } \\
\hline & anterior & posterior & anterior & posterior \\
\hline Type 1 & 17 & 10 & 16 & 9.4 \\
\hline Type 2 & 15 & 14 & 14.2 & 13.2 \\
\hline Type 3 & 16 & 9 & 15.1 & 8.5 \\
\hline Type 4 & 25 & & 23.6 & \\
\hline Total & 106 & & & \\
\hline
\end{tabular}

$34(32 \%)$ admitted with history of bleeding per vagina, further $26(24.5 \%)$ developed bleeding after admission. In $12(11.3 \%)$ cases placenta previa was diagnosed during clinical examination or cesarean delivery. 28 (26.4\%) cases stayed for more than a week in the hospital after diagnosis of placenta previa.

In the present series there were $34(32 \%)$ cases with one prior cesarean delivery, $5(4.7 \%)$ cases with 2 prior cesarean deliveries, $8(7.5 \%)$ had prior abortion and 1 $(0.9 \%)$ case had prior manual removal of placenta.

$91(85.8 \%)$ cases delivered by caesarean delivery and 15 $(14.2 \%)$ cases were delivered by vaginal route. 
$13(12.3 \%)$ cases delivered prior to 32 weeks, $29(27.4 \%)$ cases delivered between 32-37 weeks and 64 (60.3\%) cases delivered after 37 completed weeks.

Table 3: Additional surgical procedures/maneuvers carried out to control bleeding.

\begin{tabular}{|lll|}
\hline Type of procedure & Number & Percentage \\
\hline $\begin{array}{l}\text { Cervico isthmic apposition } \\
\text { stitch }\end{array}$ & 05 & 4.7 \\
\hline B-lynch stitch & 03 & 2.8 \\
\hline Uterine artery ligation & 02 & 1.9 \\
\hline $\begin{array}{l}\text { Emergency peripartum } \\
\text { hysterectomy }\end{array}$ & 05 & 4.7 \\
\hline $\begin{array}{l}\text { Uterine artery ligation } \\
\text { followed by hysteectomy }\end{array}$ & 02 & 1.9 \\
\hline $\begin{array}{l}\text { Emergency peripartum } \\
\text { hysterectomy followed by } \\
\text { internal iliac artery ligation }\end{array}$ & 01 & 0.9 \\
\hline
\end{tabular}

$13(12.7 \%)$ cases had postpartum haemorrhage and 5 (4.7\%) had adherent placenta. Additional surgical procedures/maneuvers carried out to control bleeding are shown in Table 3.

In present series total $88(83 \%)$ cases received blood and blood product transfusions. Among them 24 (22.6\%) had received one unit transfusion whereas $64(60.4 \%)$ required transfusion of more than one unit.

There were $92(86.8 \%)$ ICU admissions, $4(3.8 \%)$ cases of acute kidney injury, $1(0.9 \%)$ case of septicemia and 1 $(0.9 \%)$ maternal death in the present series. Neonatal outcome has been shown in Table 4.

Table 4: Neonatal outcome.

\begin{tabular}{|lll|}
\hline Factors & Number & Percentage \\
\hline Gestational age (maturity) & & \\
28-32 weeks & 13 & 12.3 \\
33-36 weeks & 29 & 27.4 \\
$>$ 37 weeks & 64 & 60.3 \\
\hline Birth weight & & \\
$<1.5 \mathrm{~kg}$ & 8 & 7.5 \\
$1.5-2.4 \mathrm{~kg}$ & 21 & 19.8 \\
$2.5-3.4 \mathrm{~kg}$ & 67 & 63.3 \\
$>3.5 \mathrm{~kg}$ & 10 & 9.4 \\
\hline Apgar score (<7 in 5 minutes) & 13 & 12.3 \\
\hline Nicu admissions & 32 & 30.2 \\
\hline Preterm birth & 42 & 39.7 \\
\hline Still birth & 8 & 7.5 \\
\hline Early neonatal death & 15 & 14.2 \\
\hline Congenital anomaly & 2 & 1.9 \\
\hline
\end{tabular}

\section{DISCUSSION}

Placenta previa is one of the dreaded complications in obstetrics due to its associated adverse maternal and perinatal outcome. Increasing age and number of pregnancies have been shown to be an important risk factor for placenta previa. In this study nearly one fourth of women were above 30 years of age and more than three fourth of women (79\%) were multiparas. These results are comparable with the study done by Ojha $\mathrm{N}$ et al, Wu S et al. ${ }^{12,13}$

$26.4 \%$ of women were managed by Macafee and Johnson protocol, which includes bed rest, periodic blood investigations and cross matched blood ready, watch on vaginal bleeding, frequent fetal surveillance with USG steroid prophylaxis if gestation is less than 34 weeks. It causes decrease in perinatal mortality. ${ }^{14}$

Regarding previous obstetric history $36.8 \%$ had prior cesarean delivery and $7.5 \%$ had prior history of check curettage. In a retrospective cohort study of 399, 674 women, the rate for placenta previa at second birth for women with first vaginal birth was 4.4 per 1000 births, compared to 8.7 per 1000 birth for women with cesarean section at first birth. After adjustment, cesarean section at first birth remained associated with increased risk of placenta previa (OR $1.695 \%$ CI 1.44-1.76). ${ }^{15}$

In present study $7.5 \%$ cases had history of prior abortion. In a study conducted by Ojha et al, previous history of abortions (both spontaneous and induced) have been significantly associated with up to three times risk of placenta previa. $^{12}$

In present study $85.8 \%$ cases underwent cesarean delivery, main indications being major degree placenta previa, when patient is in exanguinated state due to bleeding or for other obstetric indications. Results are comparable to a study conducted by Anand et al. ${ }^{14}$

There were 13 cases of postpartum hemorrhage cases in this study, out of which 10 were managed by conservative surgical measures like cervico isthmic apposition stitch (4.7\%), B-lynch stitch (2.8\%) and uterine artery ligation (1.9\%). 3 cases underwent emergency peripartum hysyerectomy when conservative measures failed to control bleeding. There were 5 cases of adherent placenta, all 5 underwent peripartum hysterectomy following cesarean delivery. All 5 cases of adherent placenta had prior cesarean deliveries.

Regarding maternal complications there is increased rate of postpartum hemorrhage, multiple unit blood and blood product transfusions, ICU admissions, acute kidney injury which are attributable to placenta previa. This fact is substantiated by a retrospective cohort study in Nova Scotia, Canada from 1988-1995 which had 388 cases of placenta previa. Maternal complications included postpartum bleeding (RR-1.86), hysterectomy (RR33.26), blood transfusion (RR-10.05), septicemia (RR5.55) 15 . 
Neonatal morbidity in our study was also significant. 39.7 $\%$ of our patients were delivered before 37 weeks and $30.2 \%$ of newborns were admitted to the neonatal intensive care unit. We also observed a low 1-minute Apgar score. However, the 5-minute Apgar score was improved, and only $12.3 \%$ had a score $<7$. Morbidity was more marked before 34 weeks. A population based retrospective cohort study among singleton 544, 734 mother-infant pair showed that the association between low birth weight and placenta previa is chiefly due to preterm delivery and to lesser extend to fetal growth restriction. ${ }^{16}$

There was a progressive decrease in neonatal morbidity in the form of improving Apgar scores and fewer admissions to the neonatal intensive care unit as gestation advanced was observed in the study. This is also supported by the studies done by Rosenberg $\mathrm{T}$ et al and Fiaz AS et al. ${ }^{11,17}$ Therefore, waiting until 37 weeks if patient is not bleeding could decrease neonatal morbidity in our population. However, the obstetrician must weigh the risks of neonatal prematurity against the benefits of a planned delivery.

\section{CONCLUSION}

Advancing maternal age, multiparity, prior cesarean section, and prior abortions are independent risk factors for placenta previa. An increase in the incidence of these risk factors probably contributes to a rise in the number of pregnancies complicated with placenta previa. Placenta previa remains a risk factor for various maternal complications adversely affecting maternal and perinatal outcome. The detection of placenta previa should encourage a careful evaluation with timely delivery in order to reduce the associated maternal and perinatal complications.

\section{Funding: No funding sources}

Conflict of interest: None declared

Ethical approval: The study was approved by the Institutional Ethics Committee

\section{REFERENCES}

1. Cunningham FG, Leveno KJ, Bloom SL, Haulh JC, Gilstrap LC, Wenstrom KD, editors. Obstetric haemorrhage. In: Williams Textbook of Obstetrics, 22nd ed. New York: McGraw-Hill, 20 ;809-823.

2. Martin JA, Hamilton BE, Ventura SJ. Births: Final data for 2001. National vital statistics reports. Hyattsville. National Centre for Health Statistics. 2002.
3. Crane JMG, Hof VMC, Dodds L. Neonatal outcomes with placenta previa. Obstet Gynecol. 1999;93:5413.

4. Frederiksen MC, Glassenberg R, Stika CS. Placenta previa: a 22-year analysis. Am J Obstet Gynecol. 2009;180:1432.

5. Gesteland K, Oshiro B, Henry E. Rates of placenta previa and placental abruption in women delivered only vaginally or only by cesarean section. J Soc gynecol Investig. 2004;11:208.

6. James DK, Steer PJ, Weiner CP, Gonic B, editors. Bleeding in Late Pregnancy, In: Text book of High Risk Pregnancy Management Options 3rd ed. Saunders, 59. 2010:1259-1266.

7. Salihu HM, Li Q, Rouse DJ. Placenta previa: neonatal deaths after live births in the United States. Am J Obstet Gynecol. 2003;188:1305-8.

8. Cotton D, Read JA, Paul RH, Quilligan EJ. The conservative aggressive management of placenta previa. Am J Obstet Gynecol. 1999;137:687-95.

9. Kaseem GA, Azlahrani AA. Maternal and neonatal outcomes of placenta previa and placenta acreta three years of experience with a two- consultant approach. Int J Womens Health. 2013;5:803-10.

10. Onwere C, Urganci IG, Cromwell DA, Mahmood TA, Templeton A, Jan H, et al. Maternal morbidity associated with placenta previa among women who had elective caesarean section. European J Obst Gyn Repro Bio. 2012;159:62-6.

11. Rosenberg T, Pariente G, Sergienko R, Wiznitzer A, Sheiner W. Critical analysis of risk factors and outcome in placenta previa. Arch Gynecol Obstet. 2011;284:47-51.

12. Ojha N. Obstetric factors and pregnancy outcome in placenta previa. J Institute Med. 2012;34:38-41.

13. Wu S, Kocherginsky M, Hibbard J. Abnormal placentation: twenty-year analysis. Am J Obstet Gynecol. 2005;192:1458-61.

14. Bhatt AD, Meena A, Desai MR. Maternal and perinatal outcome in cases of placenta previa. Int $\mathbf{J}$ Sci Res. 2014;3(1):299-301.

15. Urganchi GI, Cromwell AC, Edozien LC, Smith GCS, Onwere C, Mahmood TA, et al. Risk of placenta previa in second birth after birth cesarean section: a population-based study and meta-analysis. BMC Pregnancy Childbirth. 2011;11:95.

16. Ananth CV, Demissie K, Smulian JC, Vintzileos AM. Relationship among placenta previa, fetal growth restriction and preterm delivery: a population based study. Obstet Gynecol. 2001;98(2):299-306.

17. Faiz AS, Ananth CV. Etiology and risk factors for placenta previa: an overview and meta-analysis of observational studies. J Matern Fetal Neonatal Med. 2003;13(3):175-90.

Cite this article as: Sarojini, Malini KV, Radhika. Clinical study of placenta previa and its effect on maternal health and fetal outcome. Int J Reprod Contracept Obstet Gynecol 2016;5:3496-9. 